\title{
El coaching como estrategia para mejorar las habilidades de aprendizaje en el personal docente de la Universidad Nacional Toribio Rodríguez de Mendoza de Amazonas, 2015
}

\section{The coaching as a strategy to improve the learning skills in teaching personnel of the National University Toribio Rodriguez de Mendoza de Amazonas, 2015}

\author{
Manuel Antonio Morante Dávila ${ }^{1}$ y José Héctor Sandoval Caicedo ${ }^{2}$
}

\begin{abstract}
RESUMEN
El presente trabajo de investigación tiene por objetivo general describir el Coaching como estrategia que permita mejorar las habilidades en el aprendizaje del personal docente de la UNTRM para aportar en el desarrollo de la calidad educativa; asimismo entre los objetivos específicos tenemos el de elaborar un diagnóstico del desarrollo de las labores académicas del personal docente de la UNTRM, establecer las características del Coaching que puede ser aplicado en el personal docente de la UNTRM, identificar las ventajas y desventajas del Coaching en el personal docente como estrategia metodológica del proceso enseñanza aprendizaje y establecer indicadores de desempeño docente relacionados a la aplicación del Coaching. La hipótesis planteada señala que el coaching como estrategia metodológica permitirá mejorar significativamente sus habilidades de aprendizaje en los logros de un mejor rendimiento en sus labores académicas. Asimismo la presente investigación es básica; como tipo de investigación se puede considerar el estudio descriptivo, pues busca describir y explicar la hipótesis y su conexión entre la aplicación del coaching y el desarrollo de la labor docente universitaria. Los métodos de investigación a utilizar son el descriptivo, el inductivo, deductivo y analítico. La muestra para la aplicación de las encuestas está determinada por 48 docentes ordinarios de las distintas Facultades de la UNTRM.
\end{abstract}

Palabras clave: Coach, Aprendizaje, Calidad Académica

\begin{abstract}
The general objective of this research work is to describe Coaching as a strategy to improve the learning skills of UNTRM teaching staff to contribute to the development of educational quality; also among the specific objectives we have to elaborate a diagnosis of the development of the academic tasks of the teaching staff of the UNTRM, establish the characteristics of Coaching that can be applied to UNTRM teaching staff, identify the advantages and disadvantages of Coaching in the teaching staff as a methodological strategy of the learning process and establish performance indicators related to the application of Coaching Words: Coach, learning, academic quality. The hypothesis put forward points out that coaching as a methodological strategy will significantly improve your learning skills in achieving better performance in your academic work. This research is also basic; the descriptive study can be considered as a type of research because it seeks to describe and explain the hypothesis and its connection between the application of coaching and the development of university teaching work. The research methods to be used are descriptive, inductive, deductive and analytical. The sample for the implementation of the surveys is determined by 48 regular teachers from the various faculties of UNTRM.
\end{abstract}

Keywords: Coach, Learning, Academic Quality

\footnotetext{
${ }^{1}$ Profesor Asociado de la Facultad de Ciencias Económicas y Administrativas de la Universidad Nacional Toribio Rodríguez de Mendoza de Amazonas; Economista.

${ }^{2}$ Profesor Auxiliar de la Facultad de Ciencias Económicas y Administrativas de la Universidad Nacional Toribio Rodríguez de Mendoza de Amazonas; Administrador de Empresas.
} 


\section{INTRODUCCIÓN}

En el Perú nuestro sistema educativo se ha diseñado para preparar a los estudiantes para el futuro laboral en un contexto en el que un título es la garantía de un mejor trabajo porque correspondía lo que estudiabas con lo que ibas a trabajar, sin embargo el proceso de enseñanza aprendizaje al interior de las aulas universitarias ha trascendido más allá de la consecución de un título y surgen preguntas cada vez más exigentes: ¿Cómo podemos educar para la autonomía para el alumno? ¿Cuál es la nueva definición que podríamos gestar de él para que esto suceda? ¿Qué podríamos modificar o potenciar en los docentes para acompañar a descubrir, en el instruir para aprender? ¿Cuál será la diferencia para un ser humano entre yo me aprendí tal cosa o yo descubrí tal otra?; algunos investigadores consideran este proceso como el llamado "paradigma emergente", (UNESCO;1997) "en donde la persona es el eje fundamental de la educación en el mundo y que según la UNESCO las políticas pedagógicas nos muestran que los procesos de dicho paradigma se basan en "Aprender a conocer" combinando una cultura general suficientemente amplia con la posibilidad de profundizar los conocimientos en un pequeño número de materias; lo que supone además aprender a aprender para poder aprovechar las posibilidades que ofrece la educación a lo largo de la vida". "Aprender a hacer" a fin de adquirir no sólo, una calificación profesional sino, más generalmente, una competencia que capacite al individuo para hacer frente a gran número de situaciones y a trabajar en equipo. Pero, también, aprender a hacer en el marco de las distintas experiencias sociales o de trabajo que se ofrecen a los jóvenes y adolescentes, bien espontáneamente a causa del contexto social o nacional, bien formalmente gracias al desarrollo de la enseñanza por alternancia. "Aprender a vivir" juntos desarrollando la comprensión del otro y la percepción de las formas de interdependencia, realizar proyectos comunes y prepararse para tratar los conflictos respetando los valores de pluralismo, comprensión mutua y paz. "Aprender a ser" para que florezca mejor la propia personalidad y se esté en condiciones mejor obrar con creciente capacidad de autonomía, de juicio y de responsabilidad personal; con tal fin de no menospreciar en la educación ninguna de las posibilidades netas de cada individuo: memoria, razonamiento, sentido estético, capacidades físicas, aptitud para comunicar, etc.

En tal sentido la presente investigación considera la práctica del Coaching como una estrategia que permitirá fortalecer las competencias en el proceso de enseñanza aprendizaje por parte de los docentes de la Universidad Nacional Toribio Rodríguez de
Mendoza de Amazonas (UNTRM); los mismos que a través de múltiples herramientas convertirá a los docentes en mejores observadores de sí mismos y de su mundo de relaciones especialmente con los estudiantes de pregrado a fin de obtener el máximo rendimiento de sus competencias y sus habilidades, siendo prioridad la necesidad de tener nuevas habilidades que nos permitan prosperar en este entorno cambiante.

\section{MATERIAL Y MÉTODOS}

El material de estudio está conformado por los Docentes: Está representado por los docentes ordinarios de las siete Facultades con las que cuenta la Universidad Nacional Toribio Rodríguez de Mendoza de Amazonas.

La población para el presente estudio se ha considerado a los 110 docentes ordinarios con los que cuentan la Universidad en sus siete Facultades Académicas y que al menos cuentan con un mínimo de diez horas académicas; no se considerará al personal docente que se encuentra de licencia con o sin goce de remuneraciones.

Para la determinación de la muestra, se utiliza el muestreo probabilístico para poblaciones finitas.

$$
\mathrm{n}=\frac{\mathrm{N} \times \mathrm{Z} \alpha^{2} \mathrm{pxq}}{\mathrm{d}^{2} \mathrm{x}(\mathrm{N}-1)+Z \alpha^{2} \mathrm{pxq}}
$$

dónde:

$\mathrm{N}=$ Población total.

$\mathrm{Z} \alpha=1.96$ al cuadrado (grado de confianza del $95 \%)$

$\mathrm{p}=$ proporción esperada $(50 \%)$

$\mathrm{q}=$ proporción no esperada $(1-\mathrm{p}=50 \%)$

$\mathrm{d}=$ margen de error $(5 \%)$

$\mathrm{n}=$

$110 \times(1.96) 2 \times 0.5 \times 0.5$

$(0.05) 2 \times(110-1)+(1.96) 2 \times 0.5 \times 0.5$
$\mathrm{n}=86$ docentes.

Por tanto para corregir la muestra se utiliza la fórmula:

$$
\begin{aligned}
& \mathrm{n}=\frac{\mathrm{n}_{0}}{1+\mathrm{n}_{0} / \mathrm{N}} \\
& \mathrm{n}=\frac{86}{1+86 / 110} \\
& \mathrm{n}=48 \text { docentes. }
\end{aligned}
$$

Característica de la muestra la estructura organizativa a través de las líneas de autoridad y funcional.

Considerando el tipo de investigación se puede considerar el estudio descriptivo y explicativo, pues busca describir y explicar la hipótesis y su conexión entre la aplicación del coaching y el desarrollo de la labor docente universitaria. 
Descriptivo: Conoció la aplicación del coaching como estrategia metodológica.

Inductivo: Se concoció las conclusiones de hechos observables aplicando las encuestas a los docentes de la UNTRM y contar con resultados de cómo el coaching es una estrategia catalizadora en el mejor desempeño del personal docente, además de recopilar información secundaria de fuentes bibliográficas para contar con definiciones relacionadas con su desempeño académico.

Deductivo: Este método nos permitió saber, conocer conceptos, enunciados, párrafos los cuales nos permitirán llegar a conclusiones, la cual se ha realizado mediante la recopilación de información proveniente de material bibliográfico.

Analítico: Este método se fragmentó en sus partes con el fin de dar a conocer cada una de las características, cualidades, preferenctias del objetivo de estudio en este caso los docentes de la UNTRM; con la interpretación de cuadros y gráficos estadísticos producto de la tabulación aplicada, para su posterior desarrollo en el programa Excel.

\section{RESULTADOS}

Figura $N^{\circ} 01$ Satisfacción en el trabajo docente

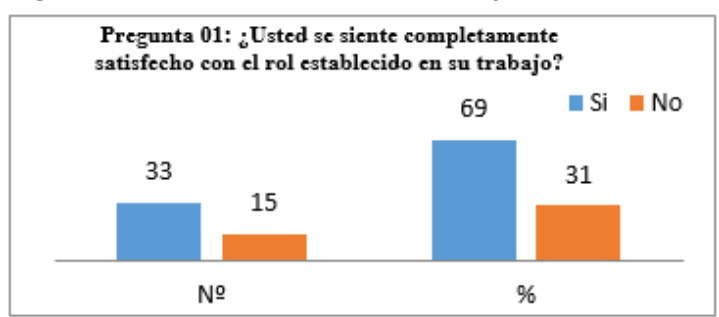

Fuente: Encuesta realizada

El $69 \%$ de los docentes encuestados se sintieron satisfechos con las funciones que realizan como docente en aula; claro está desde su punto de vista como facilitador de información para mejorar los conocimientos de los estudiantes.

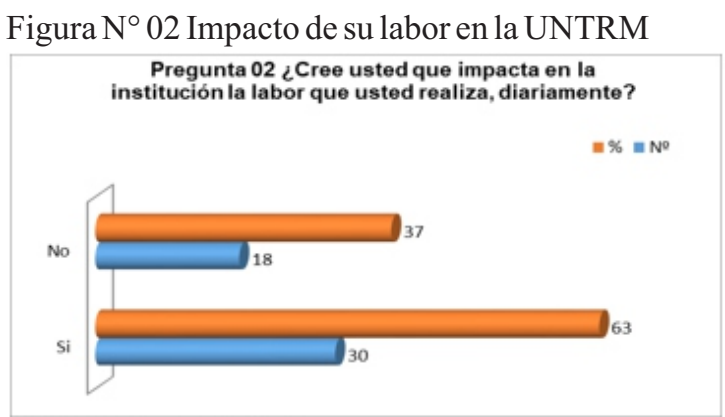

Fuente: Encuesta realizada

El 63\% de los docentes encuestados razonaron que el trabajo realizado en aulas es significativa en la imagen que tiene la Universidad en la región y a nivel nacional.

Figura $\mathrm{N}^{\circ} 03$ Juicioso de las dificultades humanas en la UNTRM

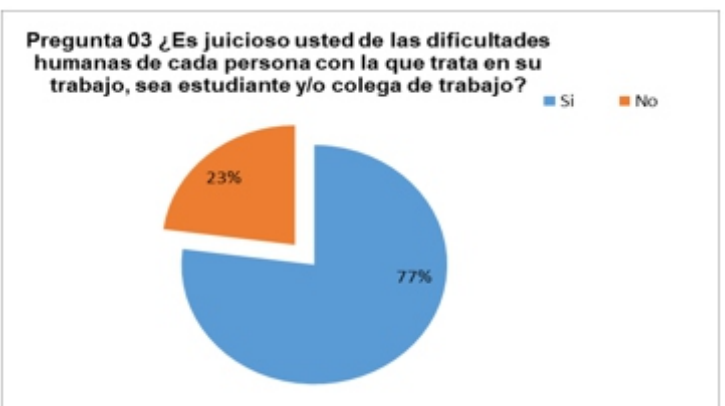

Fuente: Encuesta realizada.

Un $77 \%$ de los docentes encuestados indicaron que dialogan con los colegas y los estudiantes sobre los comportamientos en aula a fin de corregir los problemas que pudieran existir.

Figura $\mathrm{N}^{\circ} 04$ Adaptación a equipos de trabajo

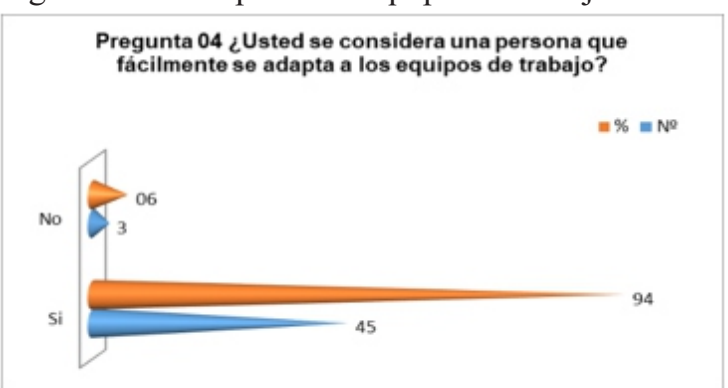

Fuente: Encuesta realizada

El 94\% de los docentes encuestados indicaron que no tienen inconvenientes para adaptarse a equipos de trabajo dentro de cada Facultad y poder cumplir con el desarrollo de sus labores académicas.

Figura $\mathrm{N}^{\circ} 05$ Actitud de servicio y atención de docentes hacia estudiantes

Figura $\mathrm{N}^{\circ} 05$ Actitud de servicio y atención de docentes hacia

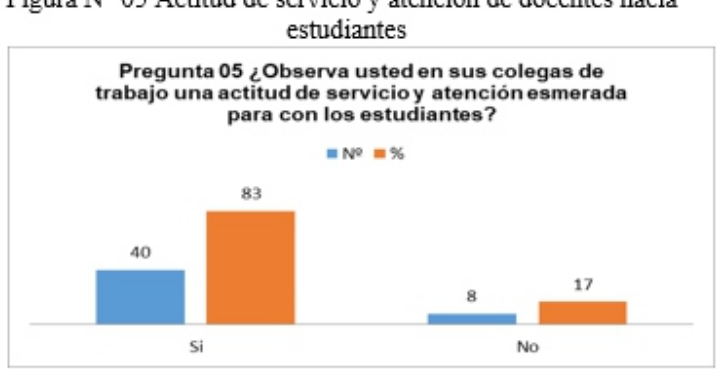

Fuente: Encuesta realizada

El 83\% de los docentes piensa que los colegas tienen esa predisposición para brindar el mejor de los esfuerzos en el desarrollo de las labores académicas en beneficio de los estudiantes. 
Figura $N^{\circ} 06$ Apoyo del Decano de Facultad a su trabajo

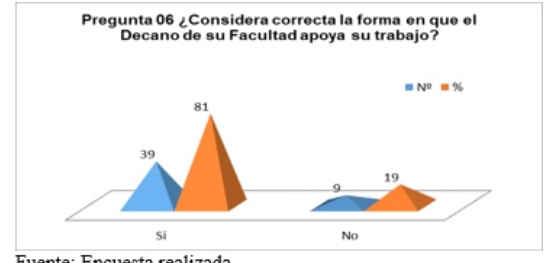

El $81 \%$ de los docentes señalan que el trabajo del Decano de la Facultad a la que brinda sus servicios académicos se encuentra dentro del apoyo ideal y que le permite sentirse augusto en el desarrollo de su trabajo.

Figura $\mathrm{N}^{\circ} 07$ Organización de proceso enseñanza aprendizaje

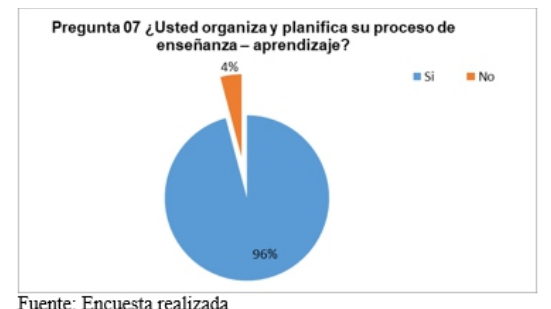

E1 96\% de los docentes encuestados si programa y planifica el desarrollo de sus labores académicas de acorde con algunas técnicas y procedimientos pedagógicos; siendo el tiempo y la falta de capacitación algunos de los impedimentos para conseguir tal fin.

Figura $\mathrm{N}^{\circ} 08$ Dificultad de estudiantes para rendir exámenes y/o evaluaciones.

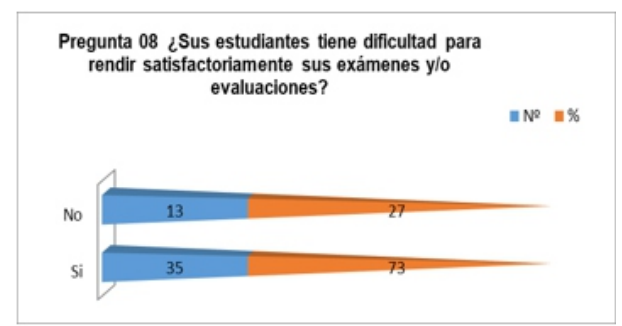

Fuente: Encuesta realizada

El $73 \%$ de los docentes encuestados consideran que los estudiantes tienen problemas significativos para poder desarrollar satisfactoriamente los exámenes y/o evaluaciones programadas.

Figura $\mathrm{N}^{\circ} 09$ Habilidades rescatables de estudiantes

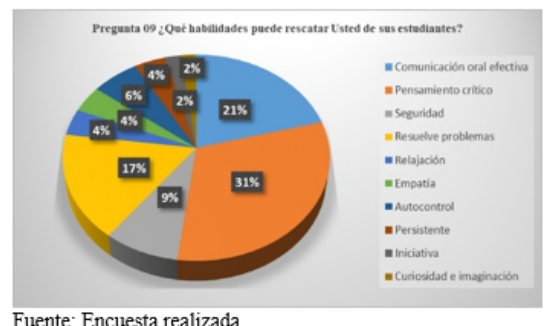

Fuente: Encuesta realizada.
El 31\% de los docentes encuestados señalan que sus estudiantes tienen la habilidad de plantear problemas referentes al tema producto del desarrollo de la clase programada pero todo ello por iniciativa del docente y no del mismo estudiante.

Figura $\mathrm{N}^{\circ} 10$ Ordena tareas a estudiantes

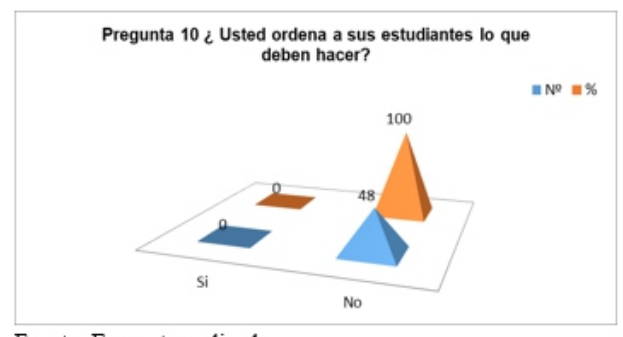

Fuente: Encuesta realizada

El 100\% de los docentes encuestados consideran que no dan órdenes a sus estudiantes para el cumplimento de las tareas a desarrollar como parte del cumplimento del avance silábico.

Figura $\mathrm{N}^{\circ} 11$ Maneras de hacer recordar conocimientos

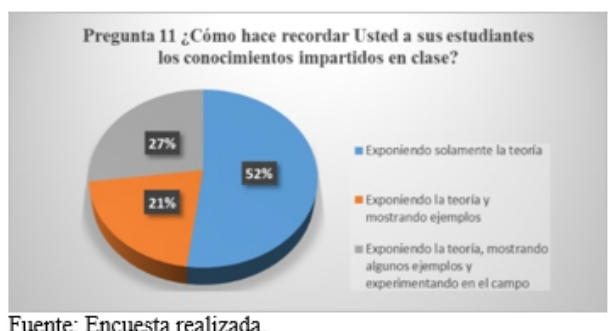

El 52\% de los docentes encuestados consideran que con la exposición de la teoría en sus respectivos cursos logran materializar un repaso en los temas y/o tópicos vistos en clase.

Figura $\mathrm{N}^{\circ} 12$ Inconvenientes para realizar preguntas

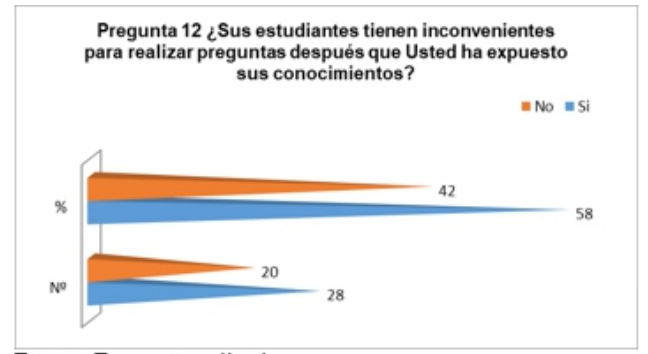

Fuente: Encuesta realizada.

El 58\% de los docentes encuestados afirman que sus estudiantes tienen inconvenientes para realizar preguntas antes, durante y después del desarrollo de una clase, siendo el temor al considerar absurda la pregunta la principal causa. 
Figura $\mathrm{N}^{\circ} 13$ Discusión sobre conocimientos expuestos en clase

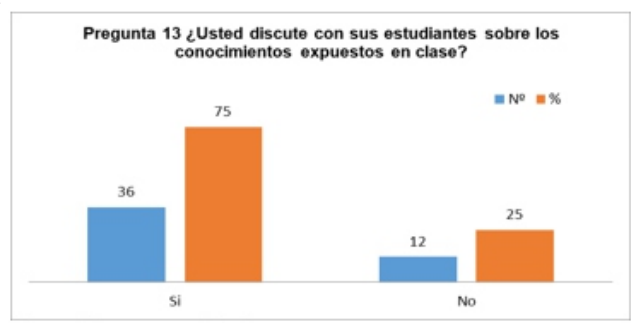

Fuente: Encuesta realizada.

Figura $\mathrm{N}^{\circ} 14$ Preguntas que no han podido absolverlas

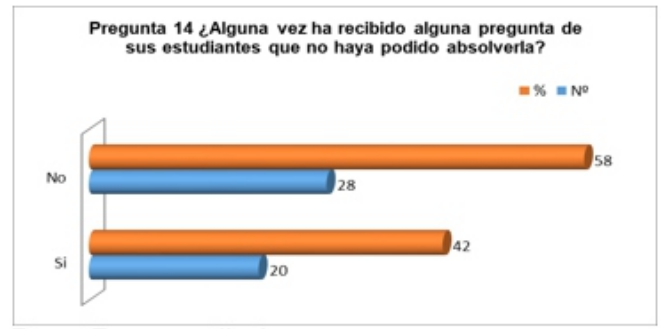

Fuente: Encuesta realizada

El 42\% de los docentes encuestados mencionan haber recibido alguna vez una pregunta de parte de sus estudiantes que no pudieron absolverá en su momento pero quedó como parte de un tema a investigar y que se trató en la siguiente clase.

Figura $\mathrm{N}^{\circ} 15$ Capacitación en docencia.

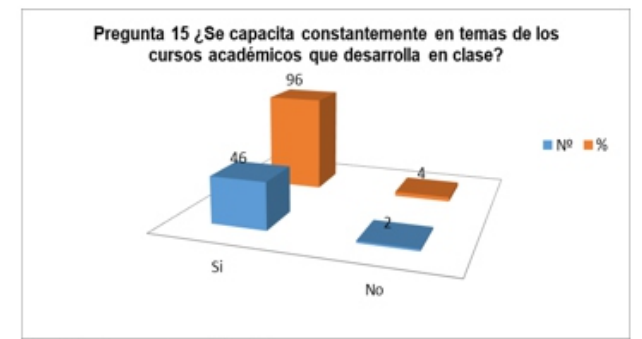

Fuente: Encuesta realizada.

El $96 \%$ de los docentes encuestados indican capacitarse en temas relacionados a los tópicos a tratar en el desarrollo de los syllabus, sin embargo consideran que no es lo suficiente para mejorar su rendimiento.

Figura $\mathrm{N}^{\circ} 16$ Resuelve ejercicios planteados por estudiantes.

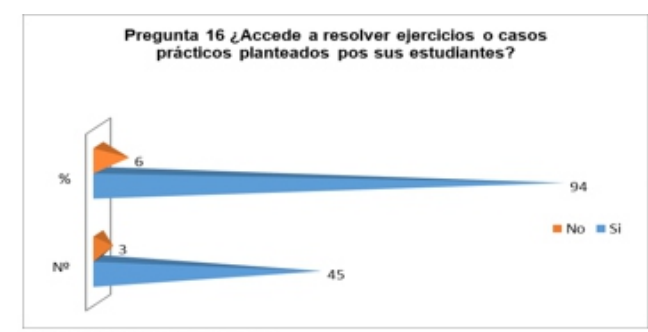

Fuente: Encuesta realizada.
El 94\% de los docentes encuestados señala resolver los ejercicios y casos prácticos que son planteados por los estudiantes para mejorar su rendimiento al momento de la evaluación correspondiente.

Figura $\mathrm{N}^{\circ} 17$ Ayuda a vencer dificultades

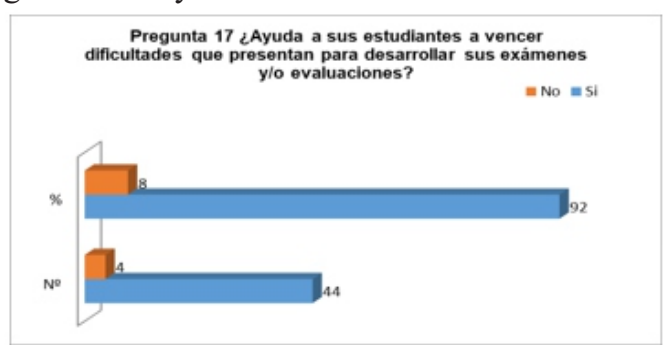

Fuente: Encuesta realizada.

El 92\% de los docentes encuestados participan de la preparación de los estudiantes al momento de programar la rendición de sus exámenes y evaluaciones programadas en los syllabus correspondientes, es decir se facilita al estudiante algunos materiales que permitan mejorar su rendimiento.

Figura $N^{\circ} 18$ Retroalimentación de clases

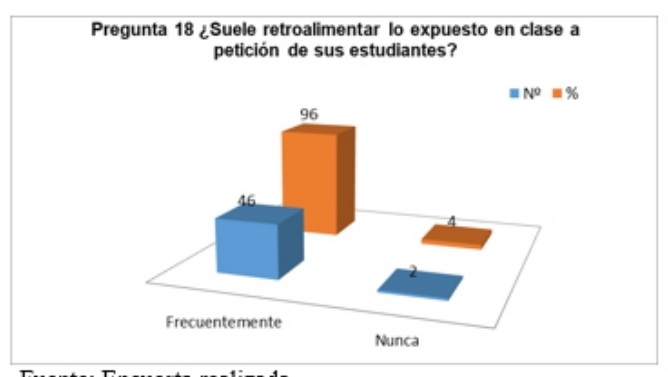

Fuente: Encuesta realizada.

El $96 \%$ de los docentes encuestados aplican la retroalimentación como técnica pedagógica para fortalecer los conocimientos aprendidos por los estudiantes a petición de los mismos.

Figura $N^{\circ} 19$ Puntualidad asistencia a clases

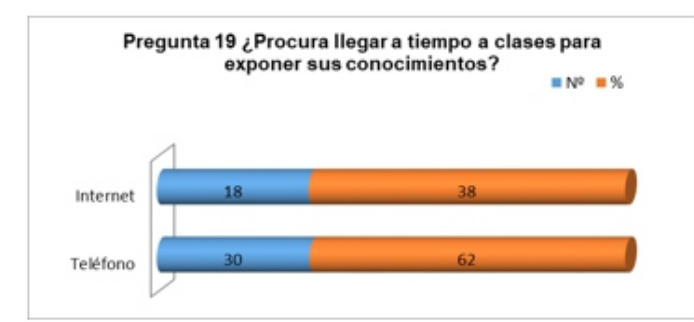

Fuente: Encuesta realizada.

El 62\% de los docentes mencionan llegar dentro del tiempo establecido para el inicio del desarrollo de sus labores académicas; motivado ello entre otras causas por la dificultad del acceso al Campus Universitario, sobre todo en las primeras horas de la mañana. 
Figura $\mathrm{N}^{\circ} 20$ Conversa sobre situaciones locales o regionales

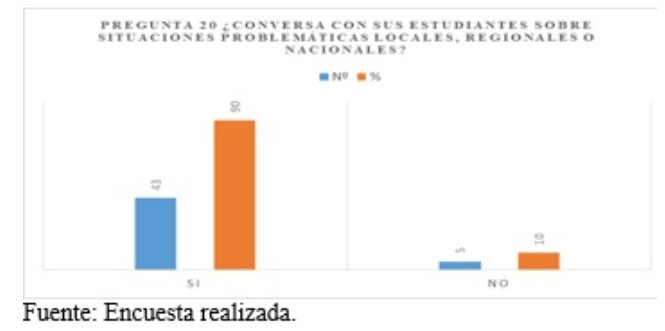

El 90\% de los docentes durante el desarrollo de sus actividades académicas propone temas relacionados con problemas sociales, políticos y de actualidad, en la cual el estudiante pueda participar activamente.

Figura $\mathrm{N}^{\circ} 21$ Temas de conversación y debates con estudiantes

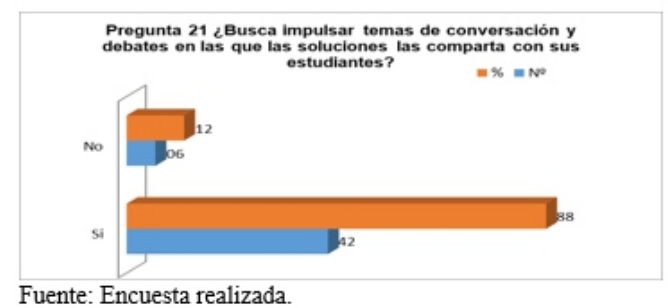

El 88\% de docentes busca que el estudiante participe de la aplicación de problemas relacionados con los temas que se encuentran en el desarrollo de los cursos aprobados y registrados en los syllabus correspondientes.

\section{DISCUSIÓN}

Es esencial que para mejorar y conseguir una educación de calidad el docente debe optimizar su desempeño al interior de las salas de clases, puesto que "la calidad de un sistema educativo tiene como techo la calidad de sus docentes" (McKinsey \& Company, 2007 p. 17). Tal es la importancia del desempeño docente que se convierte en un predictor del rendimiento de sus estudiantes y en una alternativa para mejorar los resultados de aprendizaje (Ortúzar, et al. 2010; Hunt, 2009). Es por ello que en la UNTRM es básico apostar por que las condiciones que posea un docente sean las pedagógicamente adecuadas a fin de poder conseguir rendimiento esperado en los estudiantes y esto se vea reflejado en la consecución de la inserción laboral de los mismos en el ámbito local, regional y/o nacional.

El coaching es un cierto tipo de relación que se establece entre dos (o más) personas en las que una de ellas (la llamaremos coach) facilita el éxito de la otra (la llamaremos coachee); bien, se tiene pues un coach y uno (o varios) coachee con los que esta persona interactúa en el marco de una relación a la que se llama coaching; en la UNTRM se identifica al docente de aula como coach, es decir es el facilitador de los conocimientos referidos a los temas que se encuentran en los syllabus y que son recepcionados por el coachee representado por el estudiante de las distintas facultades.

El coaching educativo se dirige a los tres estamentos principales del contexto escolar: el equipo docente, los padres y los alumnos. El objetivo es desarrollar la confianza del entrenado en sí mismo para que así tome decisiones, asuma responsabilidades consiguiendo así los objetivos o metas que se hayan propuesto al inicio del proceso. La ventaja del coach es que al no pertenecer a la comunidad educativa aparece como un profesional imparcial que observa con ojos diferentes la realidad escolar. El Coaching Educativo desea apostar por un aprendizaje individualizado para poder guiar, trasmitir y enseñar a los tres estamentos de la Educación (alumnos, formadores y padres) un nuevo modelo educacional. Al integrar esta nueva metodología los alumnos, formadores y padres serán los pilares fundamentales de una educación centrada en las personas. En la UNTRM tanto las Facultades representados por sus Decanos así como los docentes en coordinación con los estudiantes se indentifican con la propuesta de aplicación del COACHING como estrategia metodológica para mejorar el desarrollo académico y por ende el nivel de competitividad del estudiante.

Es así como el coaching se ha convertido en una herramienta que aporta y contribuye al desarrollo de estrategias que favorecen el crecimiento personal y profesional de quienes se esfuerzan por conseguir proactivamente el éxito; por lo tanto, este puede entenderse como un proceso integral que busca ayudar a las personas a producir resultados extraordinarios en sus vidas, carreras, negocios y organizaciones; a través del cual se mejora el desempeño, se profundiza en el conocimiento de sí mismos y mejora la calidad de vida, proporcionando un aprendizaje que genera transformación de comportamientos sostenidos en el tiempo, con acciones y reflexiones continuas (Lozano, 2008). Es en ese marco que los docentes universitarios de la UNTRM buscan la consecución del éxito de los estudiantes al finalizar sus estudios universitarios y su incorporación al proceso productivo a través del mercado laboral que logre mejorar el nivel competitivo del país.

Platón discrepaba en algunos aspectos ideológicos con su maestro Sócrates y sin embargo entendía el valor de las conversaciones tal como le enseñó su maestro. Los coaches, entienden que el coaching no es una ideología, sino más bien un método basado en procesos de relaciones humanas que puede trabajar con las personas independientemente de sus credos, 
religiones y filosofías personales (Ravier 2005). Es por ello que en la UNTRM puede aperturarse un espacio para introducirse el Coaching como una metodología que permita fortalecer las competencias de los docentes en el proceso enseñanza aprendizaje en sus estudiantes.

El Coaching como proceso dinámico e interactivo, y que consiste en asistir a otros en el logro de sus metas, y en el desarrollo de su propio potencial, para convertirse en mejores observadores de sí mismos y de su mundo de relaciones, con el propósito de generar nuevas posibilidades de acción (Wolk, 2013). Puede ser considerado como un proceso útil a ser aplicado en la UNTRM, pues se tienen al personal docente con actitudes para adecuarse a dicho proceso conociendo además que esto permitirá que el estudiante se sienta realizado en las labores que pueda efectuar como profesional y como ser humano.

\section{CONCLUSIONES}

- El docente de la UNTRM presenta las siguientes características como parte de su desempeño académico en aula:

Regular dominio y seguridad del contenido que se enseña, entendiendo el curriculo y sus objetivos.

Bajo conocimientos y habilidades de metodologías apropiadas y variadas.

Aceptable conocimiento por los estudiantes e interés por su aprendizaje.

Capacidad de reflexionar en las prácticas de enseñanza y las respuestas de los alumnos y la toma de decisiones a partir de ello. Capacidad de crear y sostener un clima de aprendizaje efectivo.

Predisposición y capacidad de introducir y desarrollar nuevos programas o paradigmas de enseñanza y aprendizaje.

Existencia de un buen ánimo, con compromiso por lograr los objetivos de la enseñanza.

Habilidad para motivar el aprendizaje y la comunicación efectiva.

La gran mayoría tiene buen carácter, ética y disciplina personal.

Habilidad de trabajar con otras personas y construir buenos vínculos dentro de la Universidad y la comunidad.

Las características que pueden considerarse del Coaching y que pueden ser aplicado al personal docente de la UNTRM tiene que ver con la metodología que puede materializarse en cuatro etapas y siete pasos, siendo estos:

Paso 1: Generación de contexto. Contrato -Compromisos. Aquí el docente Coach genera un contexto agradable de confianza y hace un acuerdo confidencialidad.

El Coach sugiere tomar acuerdos respecto al contexto que nos lleven a establecer un contrato.

El Coach sienta las bases para el desarrollo del Coaching y define con los participantes acuerdos básicos; es decir: frecuencia de sesiones de coaching, sesiones individuales o grupales, objetivos a desarrollar, metas, etc.

Paso 2:Acordar objetivos de proceso - fijar metas. El Coach indaga al respecto sin inducir o asumir que existe un quiebre.

Paso 3. Explorar la situación actual (realidad). Tener en cuenta los dos tipos de objetivos: Objetivo - resultado (destino final) y Objetivo proceso (forma de llegar al resultado).

Aquí se definen las pruebas que demostrarán que los has logrado.

Tener presente y en orden los recursos para lograr los objetivos (inventario).

\section{Paso 4. Reinterpretar brechas interpretativas.}

El Coach invita al Coachee a ayudarle a subir a un estrato más alto para que tenga una perspectiva diferente de la realidad, a que suma responsabilidad, ayudándolo a transformar sus juicios automáticos en explicaciones responsables, desde él mismo. Ayudarle a cambiar el análisis subjetivo de la realidad con la que la analiza el Coachee, a un análisis más objetivo de su realidad. Ayudar a generar un abanico de opciones de la realidad.

Paso 5. Diseñar acciones efectivas (Plan de acción). Finaliza este paso con la selección de aquella alternativa que el coachee considera que más le conviene para lograr el objetivo, considerando la carga de trabajo que ya tiene. ¿Qué motivaciones (beneficios) tiene para iniciar ese proceso?, ¿Con qué recursos cuenta?, ¿Qué competencias tiene para realizar esos cambios?, ¿Cuál es el costo asociado para lograr esos cambios?, ¿Cuáles son las consecuencias de implementar el plan de acción?.

Paso 6. Role-Playing. Se pueden utilizar diferentes técnicas dramáticas y emplearse en el momento en que se necesite.

Paso 7. Reflexiones finales y cierre. Aquí se 
indaga cuáles fueron los aprendizajes, cuáles son los pensamientos y sentimientos del Coachee.

¿Cuál será la forma de seguimiento del plan de acción? Avances, dificultades, consecuencias.

¿Cómo sabremos que vamos logrando el objetivo? Indicadores.

¿Cuáno te gustaría que volviéramos a reunirnos?, de acuerdo al plan de acción.

- Las ventajas del Coaching en el docente es que ello debe considerarse como una técnica de crecimiento personal que tenga como objetivo principal ayudarle a conseguir las metas que se propone y facilitar mejoras en sus competencias, conductas, habilidades y actitudes, aportándole una mejor calidad de vida como una mayor satisfacción con la práctica de su actividad profesional diaria.

- La principal desventaja del Coaching es que requiere un constante acompañamiento al estudiante y en la UNTRM el docente posee una carga horaria muy extensa, siendo esta una causal principal con este acompañamiento.

- Algunos indicadores de desempeño de los docentes en la aplicación del Coaching pueden ser:

*) Análisis de fortalezas y debilidades del alumnado como punto de partida de la asignatura.

*) Participación del alumnado en la selección de las temáticas a desarrollar en la asignatura.

*) Actividades más sencillas al principio para dar "experiencia de éxito".

*) Actividades de aprendizaje como actividades de evaluación (calidad en detrimento de cantidad).

Exposición, debate y análisis de materiales "auténticos" como metodología principal para los créditos teóricos.

Número de reuniones con los estudiantes.

\section{REFERENCIAS BIBLIOGRÁFICAS}

Bisquerra, R. (2008). Coaching: un reto para los orientadores. REOP. Vol. 19, $\mathrm{N}^{\circ} 2,2^{\circ}$ cuatrimestre, pp. 163-170.

Bou, P. (2013). Coaching para docentes. El desarrollo de habilidades en el aula. Editorial ECU.

Bradley, J. (2007); Editorial Mac Graw Hill. Bogotá.
El Coach de negocios.

Goleman, D. (1995); Inteligencia emocional. Barcelona: Kairóz.

Ravier, L. (2205). Arte y Ciencia del Coaching: su historia, filosofia y esencia.

Vitale, J. (2012). El Coach del Éxito. Ediciones Obelisco. 